\title{
Percepção e aceitação do uso da Caesalpinia ferrea como desinfetante
}

\author{
Perception and acceptance of the use of Caesalpinia ferrea as a disinfectant \\ Percepción y aceptación del uso de Caesalpinia ferrea como desinfectante
}

Recebido: 13/05/2021 | Revisado: 18/05/2021 | Aceito: 20/05/2021 | Publicado: 06/06/2021

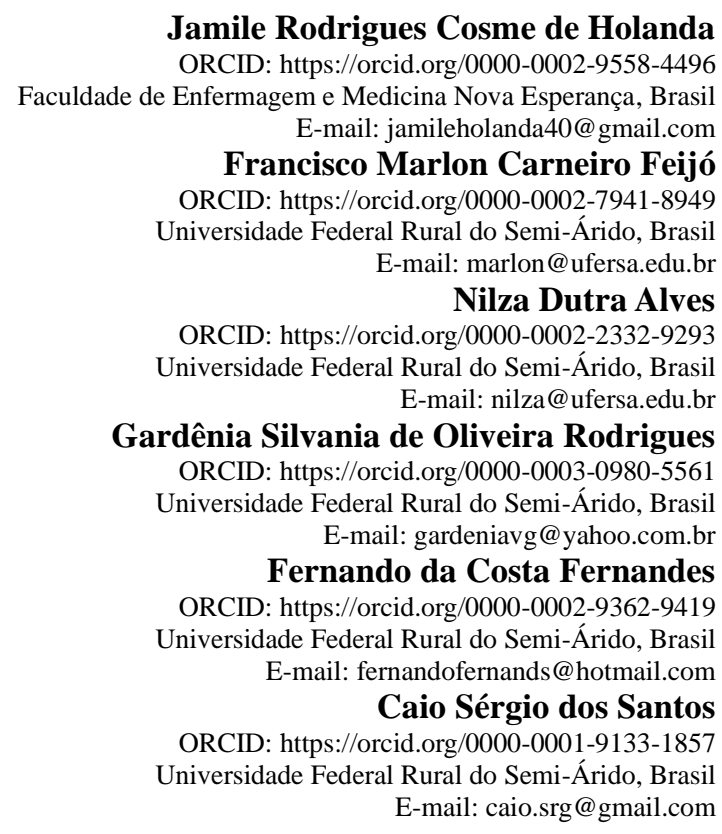

\section{Resumo}

Este trabalho objetivou verificar se há a passagem, como herança cultural, do saber sobre as plantas medicinais de geração para geração nas comunidades rurais, se os moradores das comunidades do semiárido nordestino já tinham algum conhecimento sobre as propriedades do jucá (C. ferrea), e se, baseando-se nesse conhecimento prévio e cultural, eles aceitariam usar essa planta como desinfetante natural. Para tal foi realizada entrevistas em 388 residências, onde cada residência era entrevistada somente uma pessoa, no período de outubro de 2017 a setembro de 2018, realizadas em oitos comunidades tradicionais do município de Mossoró-RN (Cordão de Sombra 1 e 2, Hipólito 1 e 2, Independência, Solidão, Cabelo de Nêgo e Três Marias). Como resultados obteve-se que 54,4\% (197) tinham idade superior a 50 anos, 64,1\% (230) eram do sexo feminino e que 77\% (278) dos entrevistados residiam na comunidade tradicional há mais de 10 anos. Quanto ao conhecimento das atividades medicinais do jucá foi observado que $81,5 \%$ (295) alegavam ter um conhecimento sobre a atividade medicinal do jucá, 53,9\% (195) afirmaram já ter utilizado o jucá em pessoas como medicina alternativa e 93,3\% (362) aceitariam utilizar o desinfetante a base do jucá. Desta forma, evidencia-se que o uso do jucá como desinfetante seria bem aceito e utilizado, pela população, em consequência de que já vem sendo utilizado para outros fins terapêuticos pelos moradores das comunidades tradicionais do entorno de Mossoró/RN.

Palavras-chave: Plantas medicinais; Jucá; Desinfecção.

\begin{abstract}
This work aimed to verify if there is a passage, as a cultural heritage, of knowledge about medicinal plants from generation to generation in rural communities, if the inhabitants of communities in the Northeastern semi-arid region already had any knowledge about the properties of the jucá (C. ferrea), and whether, based on this prior and cultural knowledge, they would accept to use this plant as a natural disinfectant. For this purpose, interviews were conducted in 388 residences, where each residence was interviewed by only one person, from October 2017 to September 2018, conducted in eight traditional communities in the municipality of Mossoró-RN (Cordão de Sombra 1 and 2, Hipólito 1 and 2, Independence, Solitude, Hair of Nêgo and Três Marias). As a result, it was found that 54.4\% (197) were over 50 years old, $64.1 \%$ (230) were female and that $77 \%$ (278) of the interviewees had lived in the traditional community for more than 10 years. Regarding the knowledge of the medicinal activities of the jucá, it was observed that $81.5 \%$ (295) claimed to have knowledge about the medicinal activity of the jucá, 53.9\% (195) stated that they had already used the jucá in people as alternative medicine and 93,3\% (362) would accept to use the disinfectant based on the jucá. Thus, it is evident that the use of jucá as a disinfectant would be well accepted and used by the population, as a
\end{abstract}


result of which it has already been used for other therapeutic purposes by residents of traditional communities around Mossoró/RN.

Keywords: Medicinal plants; Jucá; Disinfection.

\begin{abstract}
Resumen
Este trabajo tuvo como objetivo verificar si existe un pasaje, como patrimonio cultural, de conocimiento sobre plantas medicinales de generación en generación en comunidades rurales, si los habitantes de comunidades del semiárido nororiental ya tenían algún conocimiento sobre las propiedades de la jucá (C. ferrea), y si, con base en este conocimiento previo y cultural, aceptarían usar esta planta como desinfectante natural. Para ello, se realizaron entrevistas en 388 residencias, donde cada residencia fue entrevistada por una sola persona, de octubre de 2017 a septiembre de 2018, realizadas en ocho comunidades tradicionales del municipio de Mossoró-RN (Cordão de Sombra 1 y 2, Hipólito 1 y 2, Independencia, Soledad, Cabello de Nêgo y Três Marias). Como resultado, se encontró que el 54,4\% (197) tenían más de 50 años, el 64,1\% (230) eran mujeres y que el 77\% (278) de los entrevistados había vivido en la comunidad tradicional por más de 10 años. En cuanto al conocimiento de las actividades medicinales del jucá, se observó que el 81,5\% (295) afirmó tener conocimiento sobre la actividad medicinal del jucá, el 53,9\% (195) manifestó que ya había utilizado el jucá en personas como medicina alternativa y 93,3\% (362) aceptaría utilizar el desinfectante a base de jucá. Así, es evidente que el uso de jucá como desinfectante sería bien aceptado y utilizado por la población, por lo que ya ha sido utilizado con otros fines terapéuticos por vecinos de comunidades tradicionales del entorno de Mossoró/RN.
\end{abstract}

Palabras clave: Plantas medicinales; Jucá; Desinfección.

\title{
1. Introdução
}

O uso das plantas no controle de enfermidades apresenta-se como alternativa já consolidada em diversas regiões brasileiras, por se tratar de um país que possui uma grande diversidade biológica e cultural e, consequentemente, um grande conhecimento popular sobre o manejo e plantas medicinais, que perpassam de geração para geração (Costa, et al., 2018).

O uso de plantas medicinais é favorável a saúde humana, desde que se tenha conhecimento sobre sua finalidade, riscos e benefícios e seja economicamente viável. A falta de conhecimento sobre as condições de cultivo, incorreta identificação farmacobotânica, informações insuficientes sobre reações adversas e esquema posológico, podem causar efeitos adversos na saúde humana (Brasil, 2011).

O Programa Nacional de Plantas Medicinais e Fitoterápicas instituído pelo Ministério da Saúde tem como objetivo garantir à população brasileira o acesso seguro e o uso racional de plantas medicinais e fitoterápicas (Adnan, et al., 2015). Estudos de levantamentos dentro das sociedades sobre a cultura local, o cultivo, manejo, forma de utilização e outras investigações sobre plantas medicinais contribuem para o registro do conhecimento informal e consequentemente, para elaboração de pesquisas científicas que vão aumentar a gama de conhecimento sobre essas espécies vegetais, contribuindo na busca de novos fármacos (Silva \& Oliveira, 2017; Brião, et al., 2016; Sirqueira, et al., 2014).

A Caesalpinia ferrea, é uma leguminosa arbórea tropical, perenifólia ou semidecídua, de ampla dispersão e baixa densidade populacional, disseminada no Norte e Nordeste do Brasil, sendo em sua maior parte em áreas caatinga e mata atlântica, conhecida popularmente por jucá ou pau-ferro é uma das 71 espécies de plantas medicinais incluídas na lista nacional de Plantas Medicinais de Interesse para o Sistema Único de Saúde (RENISUS), com propriedades para redução de peso, tratar lesões e feridas agudas ou crônicas (cicatrizante), doenças pulmonares (tosse, asma), gripes, diabetes, anemias, diarreias, febre e inflamação. Sendo utilizados a casca, raízes, folhas e as sementes em formas de chás, decocto e extratos aquosos (Gomes, et al., 2017; Moreira \& Oliveira, 2017; Reis, Pereira \& Cansanção, 2017; Vásquez, Mendonça \& Noda, 2014; Freitas, et al., 2015; Cavalheiro, et al., 2009; Machado, et al., 2006; Thomas, Araújo \& Souza, 1998; Barros, 1982). Bem como para a construção civil e na carpintaria (Lima, et al., 2006).

A desinfecção de superfícies colabora para o controle das infecções relacionadas à assistência à saúde, por garantir um ambiente com superfícies limpas e redução no número de micro-organismos, evitando desta forma, a contaminação secundária e cruzada. Portanto, para mantê-los limpos foram criados os biocidas, ou seja, moléculas químicas ativas utilizadas para 
controlar o crescimento ou inativação de bactérias (Maillard \& Mcdonnell, 2012).

Existem muitos tipos de desinfetantes químicos no mercado, contudo, não há desinfetante que possa atuar em todas as situações e necessidades, devido à existência de diferentes condições de uso na rotina (Volkart, Spagiari \& Bizani, 2017; Brasil, 2010). Esses produtos químicos que são empregados como desinfetantes que devem ser utilizados cuidadosamente, pois muitos são nocivos aos seres humanos e ao meio ambiente, devendo ser escolhidos, manuseados e armazenados cuidadosamente, com ressalva à resistência microbiana (Reis, Pereira \& Cansanção, 2017; Brasil, 2010).

Com o avanço de novas tecnologias tornou-se possível extrair os princípios ativos das plantas medicinais, tendo como as principais formas de extração através de secagem, moagem, decocção, destilação e/ou usando um solvente extrator (Silva, Lima \& Vale, 2016). A partir dessa extração sugere-se que algumas plantas podem ser utilizadas como desinfetantes e antissépticos biológicos naturais em determinadas situações-problema em ambientes, tais como a antimicrobiana comprovada (Paiva, et al., 2015), onde o extrato bruto hidroalcóolico da casca da C. ferrea apresentou uma eficiência de $61,1 \%$ de inibição a cepas de Staphylococcus spp.

Tendo que o uso de plantas para fins medicinais é uma arte que acompanha a humanidade desde tempos remotos e que as comunidades rurais brasileiras guardam conhecimentos significativos sobre o uso das plantas medicinais (Moreira \& Oliveira, 2017; Brião et al., 2016). Tal trabalho buscou verificar se há a passagem, como herança cultural, do saber popular sobre as plantas medicinais de geração para geração nas comunidades rurais, se os moradores das comunidades do semiárido nordestino já tinham algum conhecimento sobre as propriedades do jucá ( $C$. ferrea), e se, baseando-se nesse conhecimento prévio e cultural, eles aceitariam usar essa planta como desinfetante natural.

\section{Metodologia}

Trata-se de uma pesquisa quantitativa de natureza exploratória, a qual se busca descrever sobre algo que se quer conhecer (Koche, 2011). Esta foi desenvolvida no período de outubro de 2017 a setembro de 2018 foram realizadas entrevistas em 388 residências, onde em cada moradia era entrevistada somente uma pessoa. Tais entrevistas foram realizadas nas oitos maiores comunidades tradicionais do município de Mossoró-RN (Cordão de Sombra 1 e 2, Hipólito 1 e 2, Independência, Solidão, Cabelo de Nêgo e Três Marias), conforme a localização evidenciada na Figura 1, sendo escolhidas estas comunidades por viverem de forma harmoniosa com a natureza e por contribuírem com os saberes culturais sobre as plantas medicinais.

Ressalta-se que todas as residências das comunidades foram visitadas, participando da pesquisa as que no momento disponha de uma pessoa, que se enquadrasse nos critérios de inclusão, no momento da coleta de dados, o qual aconteceu de segunda a sexta das 8 às 11 horas pela manhã e dás 13 às 17 horas no período da tarde.

Para tal utilizou-se um questionário baseado em Queiroga (2015). Os questionários foram aplicados de acordo com a metodologia de Medronhos, et al. (2008), utilizando como critérios de inclusão: ser maior de 18 anos, estar esclarecido quanto a pesquisa e ter assim assinado o Termo de Consentimento Livre e Esclarecido (TCLE). Quanto aos critérios de exclusão foram considerados: apresentar alguma incapacidade e/ou deficiência mental que prejudiquem a autonomia dos entrevistados para poder responder aos questionários, não aceitar participar da pesquisa e não assinar o TCLE, mesmo após o esclarecimento da mesma. 
Figura 1. Localização das comunidades que subsidiaram a pesquisa, localizadas nos entornos do munícipio de Mossoró/RN.

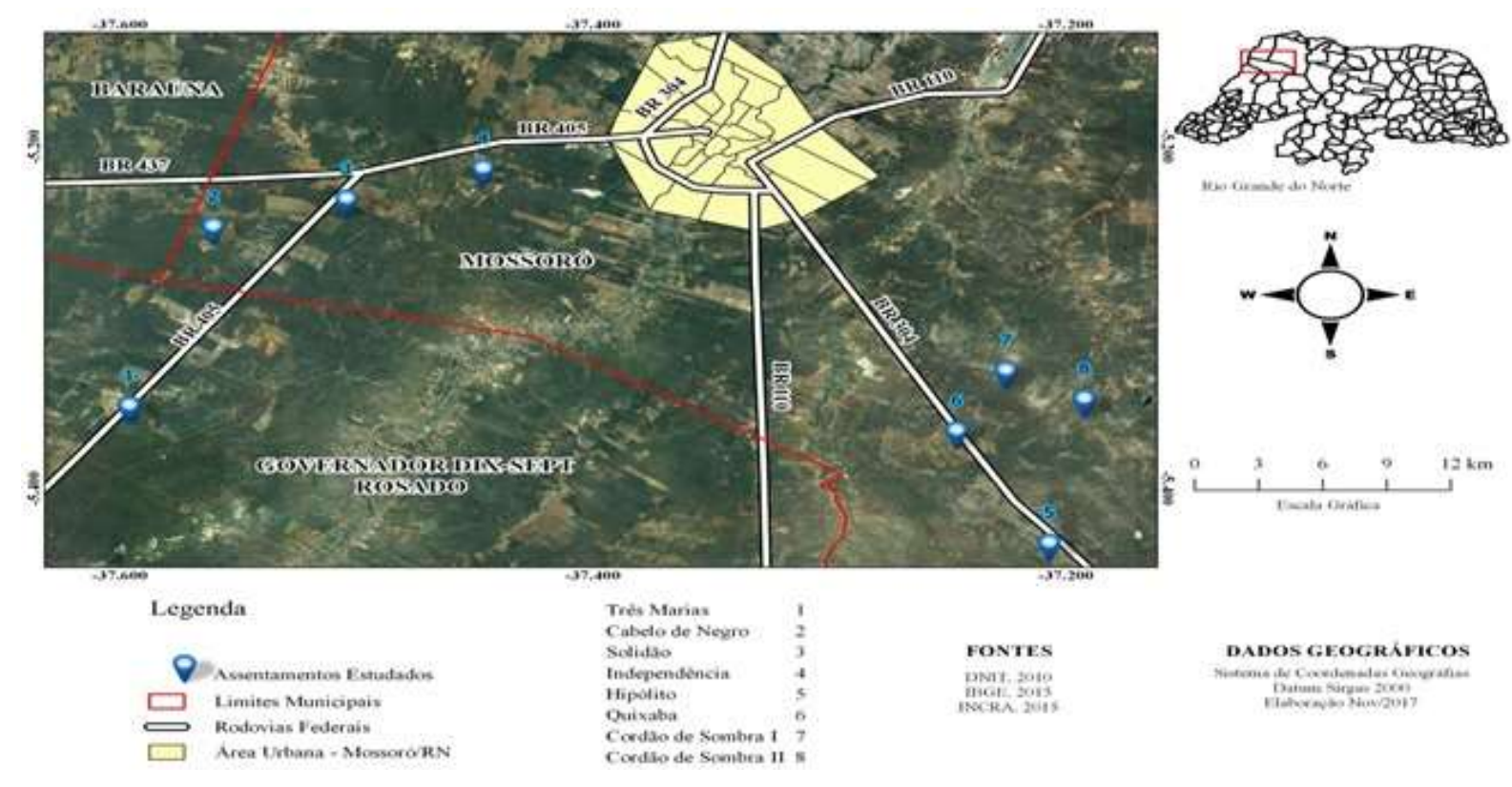

Fonte: Holanda (2019).

Para análise dos dados foi utilizado o software estatístico SPSS 23.0 (Statistical Package for he Social Sciences). A identificação dos fatores que influenciam o uso da C. ferrea (Jucá) como desinfetante foram realizadas através de Odds Ratio com respectivos intervalos de confiança a $95 \%$ e significância obtida pelo teste de Qui-quadrado ou exato de Fisher. Este último utilizado quando as frequências foram inferiores a 5.

\section{Resultados e Discussão}

Foram aplicados 388 questionários, os quais foram considerados e analisados para compor os resultados desse trabalho os dados apenas dos informantes que aceitariam utilizar o jucá ( $C$. ferrea) como desinfetante.

A caracterização dos entrevistados está evidenciada na Tabela 1, tem-se que 54,4\% (197) tinham idade superior a 50 anos, 76,5\% (273) tinha concluído apenas o ensino fundamental, 64,1\% (230) eram do sexo feminino, 69,8\% (250) eram casados e que 77\% (278) dos entrevistados residiam na comunidade tradicional há mais de 10 anos.

A predominância do sexo feminino e de maiores de 50 anos terem uma frequência maior nesta pesquisa pode se justificar pelas mulheres na zona rural serem donas de casa e estarem disponível para a aplicação dos questionários, nos horários que foram aplicados, que aconteceu em período diurno, estes se dispuseram a contribuir com a pesquisa de forma similar aos dados encontrados por Botini, et al. (2015) e Brião, et al. (2016) os quais obtiveram 90\% dos entrevistados eram do sexo feminino com uma faixa etária que variou de 23 a 87 anos, sendo que 67\% delas tinham mais de 55 anos. E por Souza, et al. (2017) e Barros, Oliveira e Abreu (2018), quanto ao grau de escolaridade, que em sua maioria (80\%) eram sem escolaridade ou possuíam ensino fundamental incompleto. 
Tabela 1. Valores de frequência simples e porcentagem do uso do jucá caso desinfetante e a classificação socioeconômica da população.

\section{Usaria desinfetante a base de}

\begin{tabular}{|c|c|c|c|c|c|c|}
\hline \multirow{3}{*}{ Variáveis } & \multicolumn{4}{|c|}{ Caesalpinia ferrea - Jucá } & \multirow{3}{*}{ OR (IC95\%) } & \multirow{3}{*}{ p-valor } \\
\hline & \multicolumn{2}{|c|}{ Sim } & \multicolumn{2}{|c|}{ Não } & & \\
\hline & Freq. & $\%$ & Freq. & $\%$ & & \\
\hline \multicolumn{7}{|l|}{ Idade } \\
\hline 18 a 30 & 49 & 13,5 & 05 & 19,2 & $0,75(0,25-2,15)$ & \multirow{4}{*}{0,428} \\
\hline 31 a 40 & 62 & 17,1 & 05 & 19,2 & $0,94(0,33-2,70)$ & \\
\hline 41 a 50 & 54 & 14,9 & 01 & 3,9 & $4,11(0,53-31,84)$ & \\
\hline Acima de 50 & 197 & 54,5 & 15 & 57,7 & 1 & \\
\hline \multicolumn{7}{|l|}{ Escolaridade ${ }^{¥}$} \\
\hline Fundamental & 273 & 76,2 & 12 & 48,0 & $4,55(1,59-13,01)$ & \multirow{3}{*}{$0,004 *$} \\
\hline Médio & 55 & 15,4 & 07 & 28,0 & $1,57(0,48-5,10)$ & \\
\hline Superior & 30 & 8,4 & 06 & 24,0 & 1 & \\
\hline \multicolumn{7}{|l|}{ Gênero } \\
\hline Masculino & 129 & 35,9 & 07 & 26,9 & $1,52(0,62-3,71)$ & \multirow{2}{*}{0,403} \\
\hline Feminino & 230 & 64,1 & 19 & 73,1 & 1 & \\
\hline \multicolumn{7}{|l|}{ Estado civil } \\
\hline Solteiro & 53 & 14,8 & 03 & 11,5 & $0,32(0,03-3,12)$ & \multirow{3}{*}{0,209} \\
\hline Casado & 250 & 69,8 & 22 & 84,6 & $0,21(0,03-1,57)$ & \\
\hline Outros & 55 & 15,4 & 01 & 3,8 & 1 & \\
\hline \multicolumn{7}{|c|}{ Há quanto tempo reside nessa localidade } \\
\hline Até 5 anos & 45 & 12,5 & 03 & 11,5 & $1,19(0,34-4,13)$ & \multirow{3}{*}{0,529} \\
\hline 6 a 10 & 38 & 10,5 & 01 & 3,90 & $3,01(0,40-23,0)$ & \\
\hline Acima de 10 anos & 278 & 77,0 & 22 & 84,6 & 1 & \\
\hline
\end{tabular}

* Significância estatística (p<0,05); OR (IC95\%): Odds Ratio (Intervalo de confiança a 95\%); ¥ Número de respondentes inferiores em virtude da ausência de dados.

Fonte: Holanda (2019).

Szerwieski, et al. (2017) e Brião, et al. (2016) relatam que as gerações mais antigas conservam o conhecimento tradicional da utilização de plantas para o tratamento da saúde, visto que conhecem uma diversidade maior de plantas úteis, devido ao saber acumulado ao longo de suas vidas, justificando os resultados relatados nesta pesquisa com $C$. ferrea, visto que, neste trabalho, a maioria dos entrevistados eram maiores que 50 anos e já ouviram falar dos benefícios e das atividades fitoterápicas do jucá.

Conforme evidenciado na Tabela 2, em que apresenta que 81,5\% (295) alegavam ter um conhecimento sobre a atividade medicinal do jucá, destes 74,7\% (222) sabiam da atividade medicinal anti-inflamatória do jucá. Os resultados encontrados com atividade anti-inflamatório do jucá foram evidenciados por Pasa, Soares e Guarim-Neto (2010), em que afirmam que as populações rurais de países em desenvolvimento têm o conhecimento tradicional sobre o uso das plantas bem vasto, pois em muitos casos, é o único recurso terapêutico disponível. Para Santos, et al. (2018) os entrevistados afirmaram usar o fruto da C. ferrea para a gripe, o qual também teve evidenciado atividade antigripal e anti-inflamatória por Reis, Pereira 
e Cansanção (2017), já Pereira e Coelho-Ferreira (2017), evidenciaram que o chá da bajem serve para infecções urinárias.

Tabela 2. Valores de frequência simples e porcentagem do uso do jucá caso desinfetante com o conhecimento da população sobre a atividade medicinal do jucá.

Usaria desinfetante a base de

Variáveis

Caesalpinia ferrea - Jucá

\begin{tabular}{cllll}
\hline \multicolumn{2}{c}{ Sim } & OR (IC95\%) & p-valor \\
& Freq. $\%$ & $\%$
\end{tabular}

\section{Tem algum conhecimento sobre atividade \\ medicinal do jucá?}

Sim

29581,5

$67 \quad 18,5$

17

65,4

$2,33(1,0-5,46)$

Não

09

34,6

$0,046 *$

Tem algum conhecimento sobre atividade

medicinal do jucá como cicatrizante?

$\begin{array}{lllllcl}\text { Sim } & 173 & 47,8 & 11 & 42,3 & 1,24(0,56-2,79) & 0,589 \\ \text { Não } & 189 & 52,2 & 15 & 57,7 & 1 & \end{array}$

Tem algum conhecimento sobre atividade

medicinal do jucá como anti-inflamatório?

$\begin{array}{ccccccc}\text { Sim } & 222 & 74,7 & 08 & 47,1 & 3,33(1,24-8,94) & 0,021^{*} \\ \text { Não } & 75 & 25,3 & 09 & 52,9 & 1 & \end{array}$

Tem algum conhecimento sobre plantas

medicinais?

$\begin{array}{lcccccc}\text { Sim } & 344 & 95,0 & 23 & 88,5 & 2,49(0,68-9,08) & 0,158 \\ \text { Não } & 18 & 5,0 & 03 & 11,5 & 1 & \\ \text { ra você plantas medicinais são: } & & & & & & \\ \text { Plantas capazes de melhorar algum problema de } & 334 & 92,3 & & & & \\ \text { saúde } & & & 24 & 96,0 & 0,70(0,10-5,41) & \\ \text { Medicamentos farmacêuticos } & 20 & 5,5 & 01 & 4,0 & 1 & 0,709 \\ \text { Não sei } & 8 & 2,2 & 0 & 0,0 & - & \\ \end{array}$

* Significância estatística $(\mathrm{p}<0,05)$; OR (IC95\%): Odds Ratio (Intervalo de confiança a 95\%); ¥ Número de respondentes inferiores em virtude da ausência de dados.

Fonte: Holanda (2019).

Tais atividades medicinais podem ser justificadas pela presença de substâncias fitoquímica já descritas do jucá, as quais já foram evidenciadas a presença de flavonoides, saponinas, taninos, alcaloides e derivados das quinonas, como também que a folha é o órgão vegetal mais ativo metabolicamente (Magalhães, et al., 2014; Wyrepkowski, et al., 2014; Sampaio, et al., 2009).

O que corrobora com os resultados evidenciados na tabela 3, em que 53,9\% (195) dos entrevistados utiliza o jucá em seu dia a dia, que seu uso se dá por motivos culturais segundo 73,4\% (146) dos entrevistados e que 84,5\% (169) usam o jucá por indicação de familiares.

Ressaltando que, o motivo cultural ao qual esta pesquisa refere-se são os valores passados entre os familiares, ou seja, que o uso do jucá nas comunidades tradicionais de Mossoró, vem sendo garantido pela passagem de informação das atividades 
medicinais dele, de geração para geração, através de diálogos com pessoas idosas, raizeiros e donas de casa que guardam essas informações, garantindo assim a perpetuação dessa informação.

Tais dados vêm corroborando com Botini, et al. (2015) e Colet, et al. (2015), que alegam que as comunidades tradicionais apresentam uma vasta farmacopeia natural, boa parte proveniente dos recursos vegetais encontrados nos ambientes naturais e estas são utilizadas por essas populações, com isso o tempo de permanência no local influencia o nível de conhecimento de uma comunidade sobre o meio, visto que tal conhecimento é repassado entre avôs, pais e filhos. Bem como Barros, et al. (2018), ressaltam que os indivíduos mais velhos, demonstraram apresentar um maior conhecimento e serem mais criteriosos quanto a detalhes de uso, formas de preparo dentre outros fatores que envolvem plantas medicinais.

Tabela 3. Valores de frequência simples e porcentagem do uso do jucá como planta medicinal.

\begin{tabular}{|c|c|c|c|c|c|c|}
\hline \multirow{4}{*}{ Variáveis } & \multirow{2}{*}{\multicolumn{4}{|c|}{$\begin{array}{c}\text { Usaria desinfetante a base de } \\
\text { Caesalpinia ferrea - Jucá }\end{array}$}} & \multirow{4}{*}{ OR (IC95\%) } & \multirow{4}{*}{ p-valor } \\
\hline & & & & & & \\
\hline & \multicolumn{2}{|c|}{ Sim } & \multicolumn{2}{|c|}{ Não } & & \\
\hline & Freq. & $\%$ & Freq. & $\%$ & & \\
\hline \multicolumn{7}{|l|}{ Usa o jucá no seu dia a dia } \\
\hline Sim & 195 & 53,9 & 12 & 48,0 & $1,26(0,56-2,84)$ & \multirow{2}{*}{0,569} \\
\hline Não & 167 & 46,1 & 13 & 52,0 & 1 & \\
\hline \multicolumn{7}{|l|}{ Usa o jucá pelo fácil acesso? } \\
\hline Sim & 64 & 32,2 & 05 & 41,7 & $0,66(0,20-2,17)$ & \multirow{2}{*}{0,533} \\
\hline Não & 135 & 67,8 & 07 & 58,3 & 1 & \\
\hline \multirow{2}{*}{\multicolumn{7}{|c|}{$\begin{array}{l}\text { Usa o jucá em razão do difícil acesso aos serviços de } \\
\text { saúde }\end{array}$}} \\
\hline & & & & & & \\
\hline Sim & 4 & 2,0 & 0 & 0,0 & - & \multirow{2}{*}{1,0} \\
\hline Não & 195 & 98,0 & 12 & 100,0 & 1 & \\
\hline \multicolumn{7}{|l|}{ Usa o jucá pelo baixo custo } \\
\hline Sim & 29 & 14,6 & 7 & 58,3 & $0,12(0,04-0,41)$ & \multirow{2}{*}{$0,001 *$} \\
\hline Não & 170 & 85,4 & 5 & 41,7 & 1 & \\
\hline \multicolumn{7}{|l|}{ Usa o jucá por motivos culturais } \\
\hline Sim & 146 & 73,4 & 10 & 83,3 & $0,55(0,12-2,60)$ & \multirow{2}{*}{0,735} \\
\hline Não & 53 & 26,6 & 02 & 16,7 & 1 & \\
\hline \multicolumn{7}{|l|}{ Quem indicou o jucá ${ }^{\ddagger}$} \\
\hline Familiares & 169 & 84,5 & 10 & 83,3 & $1,25(0,26-6,03)$ & \multirow{3}{*}{0,850} \\
\hline Vizinhos & 27 & 13,5 & 02 & 16,7 & 1 & \\
\hline Outros & 04 & 2,0 & 0 & 0,0 & - & \\
\hline
\end{tabular}

* Significância estatística (p<0,05); OR (IC95\%): Odds Ratio (Intervalo de confiança a 95\%); ¥ Número de respondentes inferiores em virtude da ausência de dados.

Fonte: Holanda (2019).

Quanto às formas de uso medicinal do jucá, tem-se que 57,8\% (115) pessoas preferem a infusão na água, 91,5\% (182) utilizam a bajem (fruto). Já quanto a forma de obtenção da planta, tem-se que 62,1\% (123) obtém na mata, conforme evidenciado na Tabela 4. Essa busca na mata, dar-se-á pelas comunidades pesquisadas serem arrodeadas por mata nativa, portanto, sendo de fácil acesso. 
Tais resultado discordam com o encontrado por Santos, et al. (2012), Colet, et al. (2015), Barros, et al. (2018) e Santos, et al. (2018), em que a principal forma de uso citada foi a utilização do chá, sendo a parte da planta mais consumida a folha, seguida da casca e fruto. Já na pesquisa realizada por Gomes, et al. (2018), Reis, Pereira e Cansanção (2017) e Pereira e Coelho-Ferreira (2017), os moradores das comunidades pesquisadas citaram que utilizavam mais o fruto (bajem) da $C$. ferrea.

Gomes, et al. (2014), Santos, Nunes e Martins (2012) e Silva, et al. (2008), demostraram, através de vários levantamentos etnofarmacológicos realizados na área de Caatinga, sobre o conhecimento e uso de plantas medicinais com ação no diabetes, que há um conhecimento sobre o potencial antidiabético da C. ferrea. E que à obtenção da planta usada como medicinal, em maioria, dava-se pelo hábito de cultivá-las nos quintais de casa, enquanto outros conseguem no mato (Barros, et al., 2018).

Tabela 4. Valores de frequência simples e porcentagem do Uso do Jucá caso desinfetante com as formas de uso do jucá como planta medicinal.

\begin{tabular}{|c|c|c|c|c|c|c|}
\hline \multirow{4}{*}{ Variáveis } & \multirow{2}{*}{\multicolumn{4}{|c|}{$\begin{array}{c}\text { Usaria desinfetante a base de } \\
\text { Caesalpinia ferrea - Jucá }\end{array}$}} & \multirow{4}{*}{ OR (IC95\%) } & \multirow{4}{*}{ p-valor } \\
\hline & & & & & & \\
\hline & \multicolumn{2}{|c|}{ Sim } & \multicolumn{2}{|c|}{ Não } & & \\
\hline & Freq. & $\%$ & Freq. & $\%$ & & \\
\hline \multicolumn{7}{|l|}{ Costuma usar o jucá como ${ }^{¥}$} \\
\hline Chá - infunde da água fervente & 08 & 4,0 & 0 & 0,0 & - & \multirow{5}{*}{0,680} \\
\hline Chá - coloca para ferver junto com água & 23 & 11,6 & 0 & 0,0 & - & \\
\hline Maceração & 52 & 26,1 & 04 & 33,3 & $0,90(0,26-3,12)$ & \\
\hline Suco & 01 & 0,5 & 0 & 0,0 & - & \\
\hline Infusão na água & 115 & 57,8 & 08 & 66,7 & 1 & \\
\hline \multicolumn{7}{|l|}{ Qual parte usa do jucá ${ }^{¥}$} \\
\hline Folha & 01 & 0,5 & 0 & 0,0 & - & \multirow{4}{*}{0,288} \\
\hline Casca & 07 & 3,5 & 0 & 0,0 & - & \\
\hline Casca e bajem & 09 & 4,5 & 02 & 16,7 & $0,25(0,05-1,30)$ & \\
\hline Bajem & 182 & 91,5 & 10 & 83,3 & 1 & \\
\hline \multicolumn{7}{|l|}{ Onde pega a planta ${ }^{¥}$} \\
\hline Quintal & 36 & 18,2 & 03 & 25,0 & $0,58(0,14-2,45)$ & \multirow{4}{*}{0,847} \\
\hline Vizinha & 38 & 19,2 & 03 & 25,0 & $0,62(0,15-2,60)$ & \\
\hline Compra & 01 &, 5 & 0 & 0,0 & - & \\
\hline Na mata & 123 & 62,1 & 06 & 50,0 & 1 & \\
\hline
\end{tabular}

* Significância estatística (p<0,05); OR (IC95\%): Odds Ratio (Intervalo de confiança a 95\%); ¥ Número de respondentes inferiores em virtude da ausência de dados.

Fonte: Holanda (2019).

Quanto ao uso do jucá, 53,3\% (192) afirmaram já ter utilizado o jucá em pessoas como medicina alternativa, e que somente 20,6\% (74) pessoas já usaram como antissépticos em animais e 1,4\% (5) indivíduos já usaram como desinfetante (Tabela 5). Aos que já utilizaram não relataram reações adversas ao uso, corroborando com as pesquisas para avaliar a ação antidiabética do extrato das folhas da C. ferrea em ratos, que demostraram que não houve reação de toxicidade, nem óbitos em nenhumas das doses aplicadas aos animais, até o final do estudo (Hassan, et al., 2015; Vasconcelos, et al., 2011), bem como na cicatrização de feridas em estudos in vivo sem causar nenhum agravo (Pereira, et al., 2016), e na alimentação de animais 
(Magalhães, et al., 2014), sem causar agravos a população que os testaram.

Tabela 5. Valores de frequência simples e porcentagem do uso do jucá caso desinfetante com o uso do jucá em pessoas, animais e como desinfetante.

\begin{tabular}{|c|c|c|c|c|c|c|}
\hline \multirow{3}{*}{ Variáveis } & \multicolumn{4}{|c|}{$\begin{array}{c}\text { Usaria desinfetante a base de } \\
\text { Caesalpinia ferrea - Jucá }\end{array}$} & \multirow{3}{*}{ OR (IC95\%) } & \multirow{3}{*}{ p-valor } \\
\hline & \multicolumn{2}{|c|}{ Sim } & \multicolumn{2}{|c|}{ Não } & & \\
\hline & Freq. & $\%$ & Freq. & $\%$ & & \\
\hline \multicolumn{7}{|l|}{ Já usou jucá em pessoas? } \\
\hline Sim & 192 & 53,3 & 11 & 42,3 & $1,56(0,70-3,49)$ & \multirow{2}{*}{0,277} \\
\hline Não & 168 & 46,7 & 15 & 57,7 & 1 & \\
\hline \multicolumn{7}{|l|}{ Já usou jucá em animais? } \\
\hline Sim & 74 & 20,6 & 03 & 11,5 & $2,0(0,58-6,81)$ & \multirow{2}{*}{0,264} \\
\hline Não & 285 & 79,4 & 23 & 88,5 & 1 & \\
\hline \multicolumn{7}{|l|}{ Já usou o jucá como desinfetante? } \\
\hline Sim & 05 & 1,4 & 02 & 7,7 & $0,17(0,03-0,91)$ & \multirow{2}{*}{0,074} \\
\hline Não & 357 & 98,6 & 24 & 92,3 & 1 & \\
\hline
\end{tabular}

* Significância estatística (p<0,05); OR (IC95\%): Odds Ratio (Intervalo de confiança a 95\%); ¥ Número de respondentes inferiores em virtude da ausência de dados.

Fonte: Holanda (2019).

Levando em consideração a importância da conservação natural das plantas medicinais, foi questionado se na comunidade tradicional havia alguma forma de conservação dessa planta, obteve-se como resultados que 91,4\% (331) dos entrevistados disseram que não existem uma forma de conservação deste na comunidade, apesar de 99,2\% (359) deles acharem importante a conservação de tal recurso natural (tabela 06). Portanto evidencia-se que os entrevistados reconhecem a importância da conservação das plantas medicinais, apesar de não o fazerem para protegê-las.

Essas plantas são uma fonte de produtos biologicamente ativos, com uma grande diversidade de estruturas e de propriedades físico-químicas e biológicas. Desta forma, quaisquer medidas tomadas para a sua conservação tende a preservar o conhecimento da utilização dos produtos naturais com a humanidade para a prevenção e tratamento da contaminação por micro-organismos (Firmo, et al., 2011), bem como a miscigenação das culturas indígenas, africanas e europeias, as quais foram influenciadoras no uso de ervas medicinais no Brasil (Souza \& Rodrigues, 2016). 
Tabela 6. Valores de frequência simples e porcentagem do uso do jucá caso desinfetante com a conservação do jucá como recurso natural.

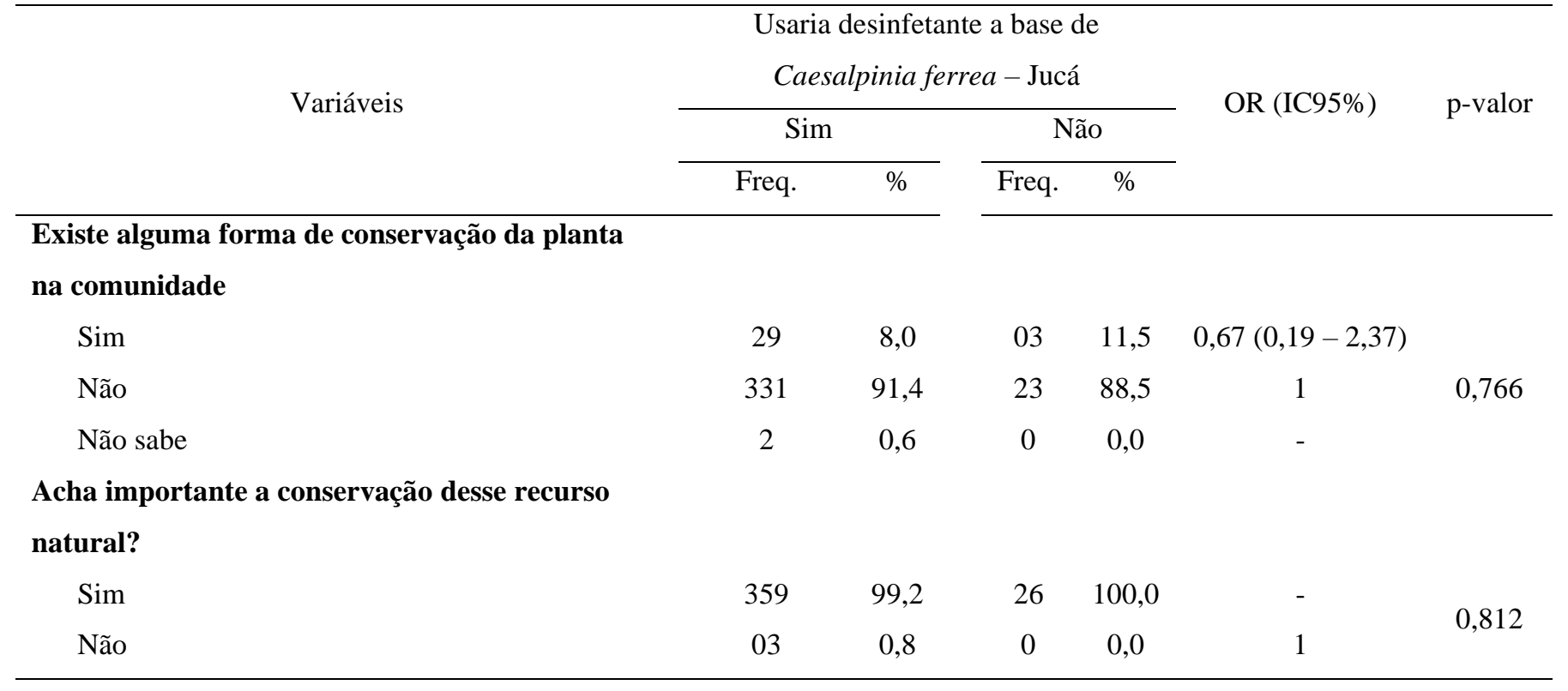

* Significância estatística (p<0,05); OR (IC95\%): Odds Ratio (Intervalo de confiança a 95\%); ¥ Número de respondentes inferiores em virtude da ausência de dados.

Fonte: Holanda (2019).

De forma geral, tem-se que dos 388 questionários aplicados 93,3\% (362) foram analisados, visto que estes aceitariam utilizar o desinfetante a base do jucá ( $C$. ferrea), caso ele já existisse, uma vez que já há a comprovação da atividade antimicrobiana por Paiva, et al. (2015), onde o extrato bruto hidroalcóolico da casca da C. ferrea apresentou uma eficiência de $61,1 \%$ de inibição a cepas de Staphylococcus spp.

\section{Conclusão}

Foi evidenciado, neste estudo, que há o repasse de informações sobre as plantas medicinais entre os familiares, de geração para geração, garantindo assim a perpetuação dessa informação. Como também se comprovou o conhecimento prévio dos moradores das comunidades tradicionais, sobre as atividades medicinais do jucá (C. ferrea).

O predomínio dessas informações pelo sexo feminino evidência que a mulher na comunidade rural exerce a atividade de dona de casa, estando mais presente na pesquisa, como também a de cuidadora da família, em virtude de obter mais informações acerca de quais plantas medicinais e ser a cuidadora responsável da família.

Por fim, este trabalho evidencia que se houvesse um desinfetante natural a base de jucá ele seria bem aceito e utilizado pela população, na medida em que já vem sendo utilizado para diversos fins, pelos moradores das comunidades tradicionais do entorno de Mossoró/RN.

Destacamos que tal pesquisa é pioneira no uso de jucá, com a possibilidade de usar seus extratos como desinfetantes. Obtendo como resultados que se existisse haveria o uso pela população. Desta forma, espera-se que esta pesquisa, contribua para outros autores iniciem a produção desse produto natural.

\section{Referências}

Adnan, M., Tariq, A., Mussarat, S., Begun, S., AbdElsalam, N. M. \& Ullah, R. (2015). Ethnogynaecological Assessment of Medicinal Plants in Pashtun's Tribal Society. BioMed Research International, 2015 (196475), 1-9. http://dx.doi.org/10.1155/2015/196475

Barros, M. A. G. (1982). Flora medicinal do Distrito Federal. Brasil Florestal, 12 (50), 35-45. 
Barros, M. S., Oliveira, Y. R. \& Abreu, M. C. (2018). Conhecimento e uso de plantas medicinais pela comunidade Cipaúba em Picos-PI. Gaia Scientia, 12 (1), 245-258. https://doi.org/10.22478/ufpb.1981-1268.2018v12n1.33348

Botini, N., Antoniazzi, C. A., Souza, K. A. \& Añez, R. B. (2015). Estudo etnobotânico das espécies Bowdichia virgilioides e Pterodon pubescens na comunidade salobra grande município de Porto Estrela, MT. Revista Biodiversidade, $14 \quad$ (2), 01-20. https://periodicoscientificos.ufmt.br/ojs/index.php/biodiversidade/article/view/2890

Brasil. (2011). Agência Nacional de Vigilância Sanitária. Formulário de Fitoterápicos da Farmacopéia Brasileira/Agência Nacional de Vigilância Sanitária. Brasília: Anvisa. p.126. From http://portal.anvisa.gov.br/documents/33832/259456/Formulario_de_Fitoterapicos_da_Farmacopeia_Brasileira.pdf/c76283eb$29 f 6-4 b 15-8755-2073 e 5 b 4 c 5 b f$

Brasil, Agência Nacional de Vigiância Sanitária (ANVISA). RDC $n^{\circ}$ 35. Regulamento Técnico para produtos com ação antimicrobiana utilizados em artigos críticos e semicríticos, de 16 de agosto de 2010. http://bvsms.saude.gov.br/bvs/saudelegis/anvisa/2010/res0035_16_08_2010.htm l\#: : text=O\%20presente\%20Regulamento\%20T\%C3\%A 9 cnico\%20tem,e\%20semicr\%C3\%ADticos\%20a\%20serem\%20comercializados.

Brião, D., Artico, L. L., Líma, L. F. P., \& Menezes, A. P. S. (2016). Utilização de plantas medicinais em um município inserido no Bioma Pampa Brasileiro. Revista da Universidade Vale do Rio Verde, 14 (2), 206-219. http://dx.doi.org/10.5892/ruvrd.v14i2.2672

Cavalheiro, M. G., Farias, D. F., Fernandes, G. S., Nunes, E. P., Cavalcanti, F. S., Vasconcelos, I. M., Melo, V. M. M., \& Carvalho, A. F. U. (2009). Atividades biológicas e enzimáticas do extrato aquoso de sementes de Caesalpinia ferrea Mart., Leguminosae. Revista Brasileira de Farmacognosia, 19 (2b), 586-591. http://dx.doi.org/10.1590/S0102-695X2009000400014.

Colet C. F., Cavalheiro C. A. N., Molin G. T. D., Gavinatto A. W., Schiavo M., Schwambach K. H., \& Oliveira K. R. (2015). Uso de plantas medicinais por usuários do serviço público de saúde do município de Ijuí/RS. Revista Brasileira de Medicina da Família e Comunidade, 10 (36), 1-13. http://dx.doi.org/10.5712/rbmfc10(36)930

Costa R. B., Camilo B. G., Toro A. M., Munhões R. A. C., Bastos E. M. V. (2018). Plantas medicinais em comunidade tradicional ribeirinha. Cuiabá - MT Biodiversidade, 17 (1), 97-103. From: https://periodicoscientificos.ufmt.br/ojs/index.php/biodiversidade/article/view/6539

Firmo W. C. A., Menezes V. J. M., Dias C. N., Alves L. P. L., Dias I. C. L., Santos Neto M. \& Olea R. S. G. (2011). Contexto histórico, uso popular e concepção científica sobre plantas medicinais. Caderno de Pesquisa, 18 (especial), 90-95. http://www.periodicoseletronicos.ufma.br/index.php/cadernosdepesquisa/article/view/746/2578

Freitas A.V.L., Coelho M.F.B., Pereira Y.B., Freitas Neto E.C. \& Azevedo R.A.B. (2015). Diversidade e usos de plantas medicinais nos quintais da comunidade de São João da Várzea em Mossoró, RN. Revista Brasileira de Plantas Medicinais, 17 (4), $845-856$. https://www.scielo.br/pdf/rbpm/v17n4s2/1516-0572-rbpm-17-4-s2-0845.pdf

Gomes D. L., Silva A. P. L., Araujo K. D., Lira E. S., Santos E. M. C. \& Costa J. G. (2018). Exploração da caatinga em assentamentos rurais do Semiárido Alagoano. Revista Ra'e Ga - O Espaço Geográfico em Análise, 45 (1), 142-152. http://dx.doi.org/10.5380/raegae

Gomes P. R. M., Firmo W. C., Vilanova C. M. (2014). Estudo etnobotânico de plantas medicinais hipoglicemiantes do bairro Maracanã no município de São Luis, Maranhão, Brasil. Scientia Plena, 10 (9), 01-11. From: https://www.scientiaplena.org.br/sp/article/view/2018/1047

Gomes T. M. F., Lopes J. B., Barros R. F. M. \& Alencar N. L. (2017). Plantas de uso terapêutico na comunidade rural Bezerro Morto, São João da Canabrava, Piauí, Brasil. Gaia Scientia, 11 (1), 253-268. http://dx.doi.org/10.21707/gs.v11.n01a17

Hassan S. K., El-Sammad N. M., Mousa A. M., Mohammed M. H., Farrag A. R. H., Hashim A. N. E., Werner V., Lindequist U. \& Nawwar M. A. E. M. (2015). Hypoglycemic and antioxidant activities of Caesalpinia ferrea Martius leaf extract in streptozotocin-induced diabetic rats. Asian Pacific Journal of Tropical Biomedicine, 5 (6), 462-471. http://dx.doi.org/10.1016/j.apjtb.2015.03.004

Holanda, J. R. C. de. (2019). Percepção do Uso e Atividade Desinfetante de Libidibia ferrea [Dissertação de Mestrado - Curso de Pós-Graduação em Ambiente, Tecnologia e Sociedade, Universidade Federal Rural do Semi-Árido, Mossoro]. Repositório Digital da UFERSA. from: https://repositorio.ufersa.edu.br/bitstream/prefix/1861/1/JamileRCH_DISSERT.pdf.

Koche, J. C. (2011). Fundamentos de metodologia científica. Petrópolis: Vozes. from: http://www.adm.ufrpe.br/sites/ww4.deinfo.ufrpe.br/files/Fundamentos_de_Metodologia_Cienti\%CC\%81fica.pdf

Lima A. C. H., Turski A. R. O., Silva B. O., Severiano J. F., Farias M. S., Silva N. R. A., Hellmann V. O., Cerqueira G. R. \& Lopes D. A. (2016). Análise da presença de microrganismos em superfícies distintas da Faculdade São Paulo de Rolim de Moura. Revista Saberes da Faculdade de São Paulo, 4 (1). $45-53$. From: https://facsaopaulo.edu.br/wp-content/uploads/sites/16/2018/05/ed5/5.pdf

Machado R. R. B., Meunier I. M. J., Silva J. A. A. \& Castro J. F. (2006). Árvores nativas para a arborização de Teresina, Piauí. Revista da Sociedade Brasileira de Arborização Urbana, 1 (1). 10-18. http://dx.doi.org/10.5380/revsbau.v1i1.66226

Magalhães I. L., Pinto F. C. L., Braz Filho R., Ferreira D. A., Lemos T. L. G. \& Monte F. J. Q. (2014). Chemical Constituents from Caesalpinia ferrea: Identification and ${ }^{1} \mathrm{H}$ and ${ }^{13} \mathrm{C}$ Resonance Assignment. American Journal of Analytical Chemistry, 5. 688-694. http://dx.doi.org/10.4236/ajac.2014.510077

Maillard J. Y \& Mcdonnell G. (2012). Selection and use of disinfectants. In Practice, 34 (4). 292-299. https://doi.org/10.1136/inp.e2741

Medronhos R. A., Bloch K. V., Raggio Luiz R. \& Werneck G. L. (2008). Epidemiologia. (2a ed.), Atheneu, 676 p.

Moreira F. R. \& Oliveira F. Q. (2017). Levantamento de plantas medicinais e fitoterápicos utilizados na comunidade quilombola - Pontinha de Paraopeba, Minas Gerais, Brasil. Revista Brasileira de Ciências da $\quad$ Vida, $\quad 5 \quad$ (5), http://jornalold.faculdadecienciasdavida.com.br/index.php/RBCV/article/view/356/244 
Paiva W. S., Souza Neto F. E., Bandeira M. G. L., Abrantes M. R., Batista A. C. L. \& Silva J. B. A. (2015). Atividade antibacteriana da casca do Jucá (Libidibia ferrea (Mart. Ex Tu.) L. P. Queiroz) frente a Staphylococcus spp. Isolados do leite de cabra com mastite. Archives of Veterinary Science, 20 (2). 141-146. http://dx.doi.org/10.5380/avs.v20i2.40422

Pasa M. C., Soares J. J. \& Guarim-Neto G. (2010). Estudo etnobotânico da comunidade de Conceição - Açu (alto da bacia do rio Aricá Açu, MT, Brasil). Acta Botanica Brasilica, 19 (2). 195-207. https://doi.org/10.1590/S0102-33062005000200001.

Pereira L. P., Mora M. R. L., Brizeno L. A. C., Nogueira F. C., Ferreira E. G. M., Pereira M. G. \& Assreuy M. A. S. (2016). Modulating effect of a polysaccharide-rich extract of Caesalpinia ferrea stem bark on wound healing of rats: role of TNF- $\alpha$, IL-1 $\beta$, NO, TGF- $\beta$. Journal of Ethnopharmacology, 187. 213-223. https://doi.org/10.1016/j.jep.2016.04.043

Pereira M. G. S. \& Coelho-Ferreira M. (2017). Uso e diversidade de plantas medicinais em uma comunidade quilombola na Amazônia Oriental, Abaetetuba, Pará. Biota Amazônica, 7 (3). 57-68. http://dx.doi.org/10.18561/2179-5746/biotaamazonia.v7n3p57-68

Queiroga, G. M. T. (2015). Plantas medicinais e fitoterápicos como alternativa terapêutica às infecções urinárias: um diagnóstico dessa realidade na saúde pública de Mossoró. Dissertação de Mestrado - Curso de Pós-Graduação em Ambiente, Tecnologia e Sociedade, Universidade Federal Rural do Semi-Árido, Mossoro]. Repositório Digital da UFERSA. From: https://ppgats.ufersa.edu.br/wp-content/uploads/sites/47/2015/03/Disserta\%C3\%A7\%C3\%A3o-GeruziaQueiroga.pdf

Reis C. R. M., Pereira A. F. N. \& Cansanção I. F. (2017). Levantamento etnobotânico de plantas medicinais utilizadas por moradores do entorno do Parque Nacional Serra Da Capivara - PI. Journal of Biology \& Pharmacy Agricultural Management, 13 (4). 7-21. http://revista.uepb.edu.br/index.php/biofarm/article/view/2924/2369

Sampaio F. C., Pereira M. S. V., Dias C. S., Costa V. C., Conde N. C. O. \& Buzalaf M. A. R. (2009). In vitro antimicrobial activity of Caesalpinia ferrea Martius fruits against oral pathogens. Journal of Ethnopharmacology, 124. 289-294. https://doi.org/10.1016/j.jp.2009.04.034

Santos L. S. N., Salles M. G. F., Pinto C. M., Pinto O. R. O. \& Rodrigues I. C. S. (2018). O saber etnobotânico sobre plantas medicinais na comunidade da Brenha, Redenção, CE. Agrarian Academy - Centro Científico Conhecer, 5 (9). 409-421. http://doi.org/10.18677/Agrarian_Academy_2018a40

Santos M. M., Nunes M. G. S. \& Martins R. D. (2012). Uso empírico de plantas medicinais para tratamento de diabetes. Revista Brasileira de Plantas Medicinais, 14 (2). 327-334. http://dx.doi.org/10.1590/S1516-05722012000200012.

Santos S. L. D. X., Alves R. R. N., Santos S. L. X., Barbosa J. A. A. \& Brasileiro T. F. (2012). Plantas utilizadas como medicinais em uma comunidade rural do semi-árido da Paraíba, Nordeste do Brasil. Revista Brasileira de Farmácia, 93 (1). 68-79. https://www.researchgate.net/profile/Jose-Barbosa47/publication/268631580_Plantas_utilizadas_como_medicinais_por_uma_comunidade_do_semiarido_da_Paraiba_Nordeste_do_Brasil/links/5c4e0e93299bf 12be3e8daad/Plantas-utilizadas-como-medicinais-por-uma-comunidade-do-semiarido-da-Paraiba-Nordeste-do-Brasil.pdf

Silva E. G., Lima D. C. S. \& Vale C. R. (2016). Avaliação do uso consciente das plantas medicinais por frequentadores de uma unidade básica de saúde de Porangatu-GO. Revista da Universidade Vale do Rio Verde. 14 (2). 975-986. http://dx.doi.org/10.5892/ruvrd.v14i2.2979

Silva J. P. A., Sampaio L. S., Oliveira L. S., Reis L. A. (2008). Plantas medicinais utilizadas por portadores de Diabetes Mellitus tipo 2 para provável controle glicêmico no município de Jequié-BA. Revista Saúde.Com, 4 (1). 10-18https://periodicos2.uesb.br/index.php/rsc/article/view/116/86

Silva T. R. \& Oliveira F. Q. (2017). Levantamento de plantas medicinais utilizadas em domicílios do bairro Maracanã, Prudente de Morais/MG. Revista Brasileira de Ciências da Vida, 5 (5). 1-22. http://jornalold.faculdadecienciasdavida.com.br/index.php/RBCV/article/view/396/231

Sirqueira B. F., Escobar Junior P. A., Lacerda G. A. \& Dasceno E. M. (2014). Estudo etnobotânico de plantas medicinais utilizadas pela população atendida no "Programa Saúde da Família" no município de Juvenília, Minas Gerais. Revista Brasileira de Pesquisa em Ciências da Saúde, 1 (2). 36-42.

Souza D. R. \& Rodrigues E. C. A. M. S. (2016). Plantas Medicinais: Indicação de raizeiros para o tratamento de feridas. Revista Brasileira em Promoção da Saúde, 29 (2). 198-203. https://doi.org/10.5020/18061230.2016.p197

Souza J. S. S., Gomes E. C., Rocha T. C. \& Bõger B. (2017). Uso de plantas medicinais por comunidades do município de Curitiba. Divers@ Revista Eletrônica Inerdisciplinar, 10 (2). 91-97. http://dx.doi.org/10.5380/diver.v10i2.54098

Szerwieski L. L. D., Cortez D. A. G., Bennemann R. M., Silva E. S. \& Cortez L. E. R. (2017). Uso de plantas medicinais por idosos da atenção primária. Revista Eletrônica de Enfermagem [Internet], 1 (1). 1-11. http://dx.doi.org/10.5216/ree.v19.42009

Thomas G., Araújo C. C., Souza P. S. (1998). Avaliação das atividades antiinflamatória, analgésica e antipirética dos extratos aquosos de Caesalpinia ferrea, Plantago major, Polygonum acre e Pterodon polygaeflorus. 10th Brazilian Symposium in Medicinal Plants, 10ed. São Paulo.

Vasconcelos C. F. B., Maranhão H. M. L., Batista T. M., Carneiro E. M., Ferreira F., Costa J., Soares L. A. L., Sá M. D. C., Souza T. P. \& Wanderley A. G. (2011). Hypoglycemic and chronic activity of Caesalpinia ferrea Martius bark extract in streptozotocin-induced diabetes in Wistar rats. Journal of Ethnopharmacology, 137 (3). 1533-1541. http://dx.doi.org/10.1016/j.jep.2011.08.059

Vásquez S. P. F., Mendonça M. S. \& Noda S. N. (2014). Etnobotânica de plantas medicinais em comunidades ribeirinhas do Município de Manacapuru, Amazonas, Brasil. Acta Amazonica, 44 (4). 457-472. http://dx.doi.org/10.1590/1809-4392201400423

Volkart P. A., Spagiari M. S. \& Bizani D. (2017). Avaliação da susceptibilidade e resistência bacteriana aos agentes controladores do crescimento de uso hospitalar e industrial. Arquivos de Ciências da Saúde da UNIPAR. 21 (1). 25-32. https://doi.org/10.25110/arqsaude.v21i1.2017.5729

Wyrepkowski C. C., Costa D. L. M. G., Sinhorin A. P., Vilegas W., Grandis R. A., Resende F. A., Varanda E. A. \& Santos L. C. (2014). Characterization and quantification of the compounds of the ethanolic extract from Caesalpinia ferrea stem bark and evaluation of their mutagenic activity. Molecules, 19 (10). 16039-16057. http;//dx.doi.org/10.3390/molecules191016039 